\title{
Observation of a Hexatic Columnar Liquid Crystal of Polydisperse Colloidal Disks
}

\author{
A. V. Petukhov, ${ }^{1}$ D. van der Beek, ${ }^{1}$ R. P. A. Dullens, ${ }^{1}$ I. P. Dolbnya, ${ }^{2}$ G. J. Vroege, ${ }^{1}$ and H. N. W. Lekkerkerker ${ }^{1}$ \\ ${ }^{1}$ Van 't Hoff Laboratory for Physical and Colloid Chemistry, Debye Institute, University of Utrecht, \\ Padualaan 8, 3584 CH Utrecht, The Netherlands \\ ${ }^{2} B M-26 B$ DUBBLE CRG/ESRF, The Netherlands Organisation for Scientific Research, \\ ESRF BP220, F-38043, Grenoble Cedex, France \\ (Received 21 February 2005; published 9 August 2005)
}

\begin{abstract}
We report the observation of a new type of columnar liquid crystal phase, which is formed by thin hard colloidal disks in a dense suspension. High-resolution small-angle x-ray diffraction reveals a combination of long-range bond-orientational order and short-range translational order between the columns, the hallmark of the hexatic phase. Our results imply that geometric frustration related to the size polydispersity of the particles destroys long-range translational order and therefore promotes the formation of this novel phase.
\end{abstract}

DOI: 10.1103/PhysRevLett.95.077801

The hexatic phase, which was first suggested as an intermediate state between a crystal and a liquid [1] in the theory of two-dimensional melting [1-4], is characterized by short-range translational order while its bondorientational order is long ranged. This paradigm was subsequently broadened to three dimensions, where 2D hexatic layers can stack into a smectic hexatic structure with interplanar bond-orientational correlations $[5,6]$. The hexatic structure can be induced by thermally excited free dislocations or by the geometric frustration between the units that constitute the system [4]. A simple example of the latter is a planar array of ball bearings of two different sizes, which retains bond-orientational order although it loses translational order [7]. Likewise, one could expect that translational ordering in polydisperse colloidal suspensions may be inhibited [8-13]. Surprisingly, colloidal platelike particles with a diameter polydispersity as high as $25 \%$ appear to form spontaneously hexagonal columnar liquid crystals $[14,15]$. Here, we demonstrate that it is the hexatic intercolumnar structure of the novel columnar phase, which allows for the accommodation of the particle polydispersity.

We used the recently developed high-resolution smallangle X-ray scattering (SAXS) setup [16] of the DutchBelgian beam line BM-26B at the ESRF (Grenoble, France). The instrumental resolution function had a full width at half maximum of $\Delta_{\text {hor }}=0.0008 \mathrm{~nm}^{-1}$ and $\Delta_{\text {ver }}=0.0015 \mathrm{~nm}^{-1}$ in the horizontal and vertical directions, respectively. To fully exploit the capabilities of SAXS, samples with large oriented domains are necessary $[16,17]$. In suspensions of hard spheres the addition of a depleting polymer at concentrations close to phase separation is known to promote the formation of large single crystals $[16,17]$. Following this scheme, we added the nonadsorbing polymer (poly-dimethylsiloxane, $M_{w}=$ $423 \mathrm{kDa}$ ) at a concentration of $0.8 \mathrm{~g} / 1$ to a suspension of sterically stabilized colloidal gibbsite platelets (with diameter $D=237 \pm 49 \mathrm{~nm}$ and a thickness of $L=18 \pm$ $3 \mathrm{~nm}$ ) in toluene. Suspensions were placed in flat capilla-
PACS numbers: 61.30. $-\mathrm{v}$, 61.10. $-\mathrm{i}$, 64.70.Md, 82.70.Dd

ries (internal cross section $0.3 \times 3 \mathrm{~mm}^{2}$ ) and stored upright for the period of at least 7 months to allow for the establishment of a gravity-controlled profile of the osmotic pressure [18]. Figure 1 shows the three-phase equilibrium of the isotropic, nematic, and columnar phases as observed between crossed polarizers [Fig. 1(a)]. The columnar phase is clearly identified by strong Bragg reflections of visible light [Figs. 1(c) and 1(d)].

Typical SAXS patterns at normal incidence are presented in Fig. 2 along with the corresponding averaged radial profiles. The isotropic phase [Fig. 2(a)] yields a pattern with a monotonically decaying scattering intensity $I$ as a function of the momentum transfer $q$ whereas the

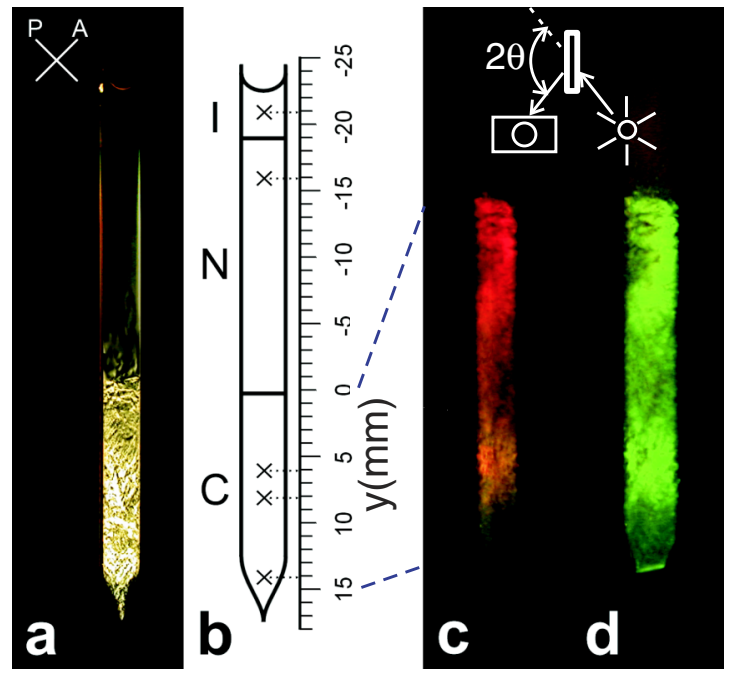

FIG. 1 (color online). Panel (a) depicts the sample between crossed polarizers (whose orientation is indicated in the left top) and panel (b) identifies the isotropic (I), nematic (N), and columnar (C) phase. Crosses mark the positions in the sample where SAXS patterns depicted in Fig. 2 were taken. Panels (c) and (d) show close-ups of the columnar phase, capturing the Bragg reflections at two different Bragg angles of $2 \theta=140^{\circ}$ (c) and $2 \theta=120^{\circ}(\mathrm{d})$. 


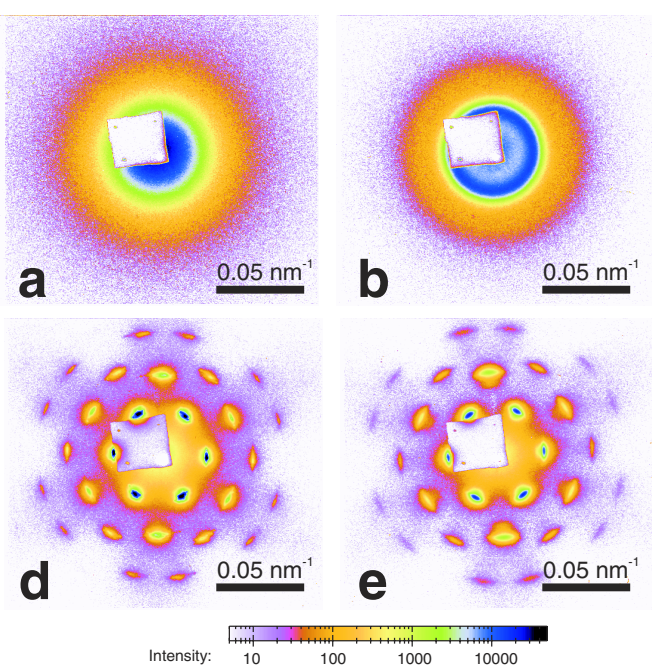

nematic phase [Fig. 2(b)] displays a broad peak in $I(q)$, which reflects the liquidlike side-to-side translational correlation between the neighboring particles. In the top part of the columnar phase [Fig. 2(c)] we observe sharp rings with a ratio of the radii of $1: \sqrt{3}: \sqrt{4}: \sqrt{7}$ that arise from the (10), (11), (20), and (21) Bragg reflections of a powder of hexagonal columnar crystals. In the lower part [Figs. 2(d) and 2(e)] a single-domain pattern with sixfold symmetry is found. This single domain extends down to the bottom of the capillary and yields very similar patterns throughout its $3 \times 8 \mathrm{~mm}^{2}$ region. As demonstrated below, in Fig. 2 the $\mathrm{x}$-ray beam is parallel to the average column direction and therefore reveals the $2 \mathrm{D}$ intercolumnar structure.

To probe the structure in the third dimension, diffraction patterns were measured in the sample turned around the vertical axis by an angle $\psi$. Only minor modifications of the pattern were observed at $|\psi|$ up to $10^{\circ}-20^{\circ}$. For larger angles the intensity of the off-axis intercolumnar reflections decays monotonically for both positive as well as negative values of the rotation angle $\psi$. The $q$ value and the width of the off-axis intercolumnar reflections do not change upon rotation, i.e., the reflections have an arc shape rather than a rod shape in the third dimension. This indicates a significant variation of the averaged column direction within the irradiated area. The texture seen in the columnar phase [Fig. 1(a)] is apparently induced by the random variation of the average column direction, without which the bottom of the capillary should appear dark since light would propagate along the optical axis.

The bending of the columnar net also allowed us to access the intracolumnar structure at a rotation angle of $70^{\circ}$ as illustrated in Fig. 3. Both the single-domain and the multidomain region exhibit a strong $(0,0)$ Bragg rod typical for a liquid crystal columnar structure with a broad peak at larger scattering angles (at $q \approx 0.27 \mathrm{~nm}^{-1}$ ), which is similar to the peak originating from the fluid face-to-face structure of the nematic. This confirms the 1D liquid intracolumnar structure.
The results presented in Figs. 2 and 3 clearly demonstrate coexistence of isotropic, nematic, and columnar phases within one capillary, which is induced by the osmotic pressure gradient due to gravity [18]. Except for the isotropic phase, the platelet orientation is (nearly) parallel to the flat walls throughout the capillary. The columnar phase consists of a multidomain region on top and a large $3 \times 8 \times 0.3 \mathrm{~mm}^{3}$ single columnar domain at the bottom.

One may think that the only difference between the multidomain and the single domain is the crystal size while their structure is equivalent. However, a careful inspection of the SAXS data reveals that this is not the case. First of all, we find a remarkable difference in the effect of gravitational compression. The intercolumnar spacing hardly changes in the multidomain region, whereas the single domain can be compressed much easier (Fig. 4). This is

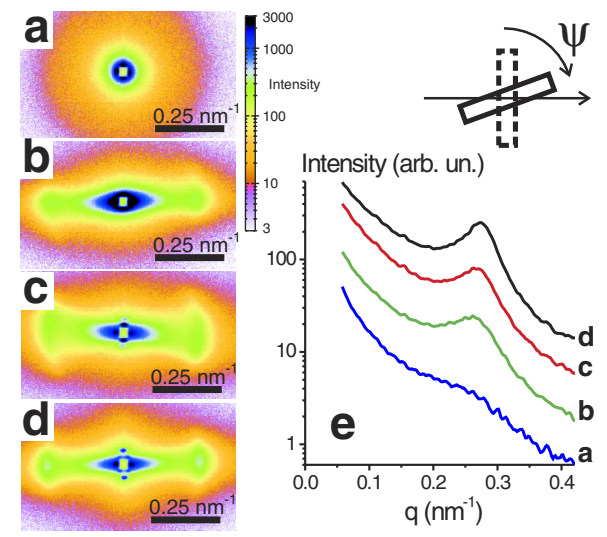

FIG. 3 (color online). SAXS data obtained after sample rotation by an angle $\psi=70^{\circ}$. Panels (a)-(d) present broadview SAXS patterns measured with $13 \mathrm{keV}$ x-rays in isotropic [(a), $y=-21 \mathrm{~mm}]$ and nematic [(b), $y=-16 \mathrm{~mm}]$ phase, multidomain [(c),y=6 mm] and single domain [(d),y= $14 \mathrm{~mm}$ ] columnar phase. Panel (e) displays the horizontal profiles of the scattering intensity in (a)-(d). The inset illustrates sample rotation (seen from top). 


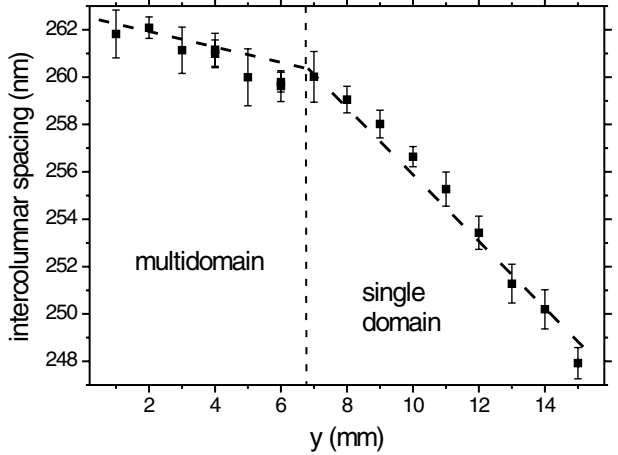

FIG. 4. The intercolumnar spacing $a$ (as influenced by gravitational compression) as a function of height $y$. The error bars represent the spread in the intercolumnar spacing as determined from the magnitude of the momentum transfer $q$ for variousorder reflections in the diffraction patterns. The vertical dashed line denotes the position of the multidomain-single-domain interface while the slanted lines are meant to guide the eye.

surprising since the compressibility of a colloidal crystal usually diminishes with increased packing fraction.

Furthermore, there is a clear difference in the width of the reflections [Figs. 5(a) and 5(b)]. To quantify that in more detail, we have studied the intensity profiles in the horizontal direction, where the resolution of the setup is higher [Fig. 5(c)]. The diffraction rings in the powder consist of sharp circular reflections of different crystallites with an apparent full-width-at-half-maximum (FWHM) of about $\delta_{\text {app }}=0.0008 \mathrm{~nm}^{-1}$, i.e., fully governed by the instrument resolution $\Delta_{\text {hor }}$, which was determined from the width of the direct beam recorded without beamstop. Surprisingly, in the single domain the radial profile of the (10) reflection clearly broadens beyond the resolution. The broadening implies that in the single crystal the translational order does not extend over the whole crystal as it should in an ideal hexagonal columnar crystal $[19,20]$, but over a range that is significantly shorter than the size of the crystallites in the multidomain region. Moreover, the (20) reflection of the single domain is further broadened significantly while that of the multidomain region remains sharp. This strongly indicates that the single crystal does not possess an average lattice [21], but gradually loses its translational order. This observation excludes a mosaic structure (consisting of small well-ordered crystallites with orientational but no positional correlations between them) as the origin of peak broadening.

Similar analysis of the reflection width was performed for patterns measured at other positions within the columnar phase. In Fig. 5(d), the right-hand axis yields an indication of the intrinsic reflection width $\delta_{\text {intr }}$ assuming that the apparent width $\delta_{\text {app }}$ is given by $\delta_{\text {app }}^{2}=\Delta_{\text {hor }}^{2}+\delta_{\text {intr }}^{2}$. The powderlike diffraction rings at $0<y \leq 6 \mathrm{~mm}$ have a radial width very close to the instrument resolution $\Delta_{\text {hor }}$, although with a certain spread of the values. The latter can be related to experimental inaccuracies as well as to effects of the finite size of the crystallites in the powder. No pronounced dependence of the peak width on the diffraction order can be seen. At $y>6 \mathrm{~mm}$, however, the (10) and, especially, (20) peaks clearly broaden with increasing $y$. For comparison, the lines in Fig. 5(d) were drawn assuming a linear increase of the intrinsic width $\delta_{\text {intr }}(y)$ at $y>6 \mathrm{~mm}$. It is also assumed that the intrinsic width of the (20) reflection is 2 times larger than that of the (10) peak.

Even stronger broadening can be seen in the azimuthal direction [Fig. 5(b)], which implies variation of the bond orientations. Also, the predominant orientation of the hexagonal bonds slowly varies on the scale of several $\mathrm{mm}$
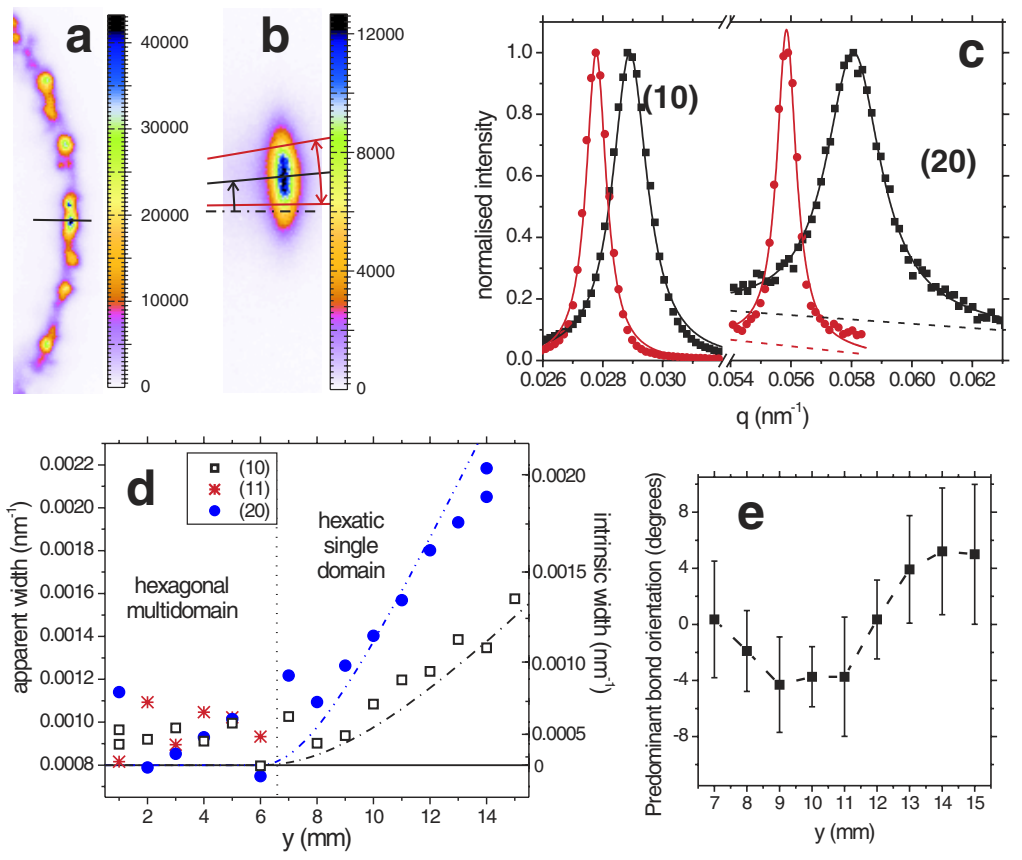

077801-3
FIG. 5 (color online). Panels (a) and (b) show close-ups of the areas of the (10) reflections in the diffraction patterns of the multidomain $(y=6 \mathrm{~mm})$ and single domain $(y=14 \mathrm{~mm})$, respectively. Radial profiles through the (10) and (20) reflections in multidomain (red circles) and hexatic domain (black squares) are shown in panel (c). The solid lines are fits to a Lorentzian profile plus a linear function (dashed lines) accounting for the background level. Panel (d) displays the radial FWHM of the (10), (11), and (20) reflections as a function of height $y$. Panel (e) shows predominant azimuthal bond orientation [indicated by the black single-headed arrow in (b)] as a function of $y$ within the hexatic domain. The vertical bars display FWHM of the reflections in the azimuthal direction [indicated by the red double-headed arrow in (b)]. 
[Fig. 5(e)]. Yet, the bond-orientational order is remarkably well preserved over macroscopic distances. Within the irradiated sample area of $0.25 \times 0.25 \mathrm{~mm}^{2}$, the bonds exhibit an average variation of $\pm 3.5^{\circ}$, whereas throughout the $3 \times 8 \mathrm{~mm}^{2}$ single domain, the overall bondorientational variation is only slightly higher: $\pm 5.2^{\circ}$ as was found by taking into account both the azimuthal broadening of every reflection and the variation of the predominant bond orientation from one pattern to the other.

The observed quasi-long-range bond-orientational order and the fast decay of the translational correlations in the single domain strongly resemble the properties of the hexatic phase. Our system consists of 1D columns, which twodimensionally pack into a $(1+2) \mathrm{D}$ hexatic structure. This structure is distinctly different from the $(2+1) \mathrm{D}$ hexatic smectic liquid crystals, which consist of 2D hexatic layers stacked one dimensionally. Some indications have been reported of the existence of a $(1+2) D$ hexaticlike structure in columnar crystals of polydisperse DNA molecules [22] and of oil tubes under shear [23] although with rather weak bond-orientational order. In these systems composed of long building blocks inducing strong longitudinal correlation, a mechanism involving dislocation loops or strings might be responsible for the occurrence of hexatic order [4,24]. In our discotic system we now find strong hexaticlike order without an intrinsically strong longitudinal correlation within each column as in Refs. [22,23]. To our knowledge, up to now no hexatic features were reported in any molecular discotic liquid crystals.

We propose that for our colloidal disks the need for accommodation of polydispersity at high compression leads to the formation of the hexaticlike columnar phase. Close to the nematic-columnar transition the system forms a powder consisting of true long-range-ordered columnar crystallites, where the relatively large free space between the columns allows for the accommodation of rather highly polydisperse particles. In contrast, in the lower part, due to gravitational compression, very little space is available. The geometrical frustration induced by the particle polydispersity can suppress the ordering upon increasing density and favor hexaticlike structuring [4,7,25]. The observed reduction of the translational correlation length [Fig. 5(d)] towards the bottom may also be an explanation for the higher compressibility of the hexatic structure. On the basis of the abrupt transition between the diffraction patterns as well as the break in the compressibility, we argue that the multidomain and single domain are in fact distinct phases: a true hexagonal and a hexatic columnar liquid crystal, respectively. The difference in the crystal size between the hexagonal columnar and the hexatic columnar phase can be related to the structural flexibility of the latter. It also explains the relative easiness [14] of crystallization of discotic colloids and allows for growth of large domains [15] out of highly diverse building blocks.
We profited from enlightening discussions with Patrick Davidson, Willem Kegel, Tanja Schilling, and Alfons van Blaaderen. Assistance of Job Thijssen, Martijn Oversteegen, Dirk Aarts, Alex de Beer, Dominique Thies-Weesie, and Xinyi Xian during experimental sessions at the ESRF is gratefully acknowledged. The Netherlands Organisation for Advancement of Research is thanked for granting us the beam time and the staff of BM-26 beamline for support.

[1] B. I. Halperin and D. R. Nelson, Phys. Rev. Lett. 41, 121 (1978).

[2] J. M. Kosterlitz and D. J. Thouless, J. Phys. C 6, 1181 (1973).

[3] A. P. Young, Phys. Rev. B 19, 1855 (1979).

[4] For an extensive overview, see D. R. Nelson, Defects and Geometry in Condensed Matter Physics (Cambridge University Press, Cambridge, England, 2002).

[5] R. Pindak et al., Phys. Rev. Lett. 46, 1135 (1981).

[6] W. H. de Jeu, B. I. Ostrovskii, and A. N. Shalaginov, Rev. Mod. Phys. 75, 181 (2003).

[7] D. R. Nelson, M. Rubinstein, and F. Spaepen, Philos. Mag. A 46, 105 (1982).

[8] P. N. Pusey, in Les Houches, Session L1, Liquids, Freezing and Glass Transitions (North-Holland, Amsterdam, 1991).

[9] P. G. Bolhuis and D. A. Kofke, Phys. Rev. E 54, 634 (1996).

[10] S. Auer and D. Frenkel, Nature (London) 413, 711 (2001).

[11] Z. Dogic and S. Fraden, Phil. Trans. R. Soc. A 359, 997 (2001).

[12] P. Sollich, J. Phys. Condens. Matter 14, R79 (2002).

[13] M. Fasolo and P. Sollich, Phys. Rev. Lett. 91, 068301 (2003).

[14] F. M. van der Kooij, K. Kassapidou, and H. N.W. Lekkerkerker, Nature (London) 406, 868 (2000).

[15] D. van der Beek et al., Eur. Phys. J. E 16, 253 (2005).

[16] A. V. Petukhov et al., Phys. Rev. E 69, 031405 (2004).

[17] A. V. Petukhov et al., Phys. Rev. Lett. 88, 208301 (2002); 90, 028304 (2003).

[18] S. Hachisu and K. Takano, Adv. Colloid Interface Sci. 16, 233 (1982); H. H. Wensink and H. N. W. Lekkerkerker, Europhys. Lett. 66, 125 (2004); D. van der Beek, T. Schilling, and H. N. W. Lekkerkerker, J. Chem. Phys. 121, 5423 (2004).

[19] R. Peierls, Surprises in Theoretical Physics (Princeton University, Princeton, NJ 1979).

[20] J. Prost and P.G. De Gennes, The Physics of Liquid Crystals (Oxford University, Oxford, 1995).

[21] A. Guinier, X-Ray Diffraction in Crystal, Imperfect Crystals, and Amorphous Bodies (Dover, New York, 1994).

[22] H. H. Strey et al., Phys. Rev. Lett. 84, 3105 (2000).

[23] L. Ramos and F. Molino, Phys. Rev. Lett. 92, 018301 (2004).

[24] S. Jain and D. R. Nelson, Phys. Rev. E 61, 1599 (2000).

[25] R. P. A. Dullens and W. K. Kegel, Phys. Rev. Lett. 92, 195702 (2004); Phys. Rev. E 71, 011405 (2005). 